\title{
Proceedings of the 23rd International Stroke Genetics Consortium Workshop: Kyoto, Japan
}

Neurol Genet 2019;5:e324. doi:10.1212/NXG.0000000000000324

\section{23rd Workshop of the International Stroke Genetics Consortium, April 12-13, 2018, Kyoto, Japan}

Yoichiro Kamatani, $\mathrm{MD}, \mathrm{PhD}^{1,2,3}$ and Stéphanie Debette, $\mathrm{MD}, \mathrm{Ph} \mathrm{D}^{2,4,5}$

${ }^{1}$ Kyoto-McGill International Collaborative School in Genomic Medicine, Kyoto University Graduate School of Medicine; ${ }^{2}$ Center for Genomic Medicine, Kyoto University Graduate School of Medicine, Kyoto; ${ }^{3}$ Laboratory for Statistical Analysis, RIKEN Center for Integrative Medical Sciences, Yokohama, Japan; ${ }^{4}$ University of Bordeaux, Bordeaux Population Health Research Center, Inserm U1219; and ${ }^{5}$ Memory Clinic, Department of Neurology, Bordeaux University Hospital, Bordeaux, France.

The 23rd Workshop of the International Stroke Genetics Consortium (ISGC) was held on April 12-13 in Kyoto, Japan, and was hosted by Yoichiro Kamatani, Stéphanie Debette, Paul Nyquist and John Cole. The ISGC is an international collaboration of physicians and scientists who have agreed to pool resources and expertise in an effort to unravel the genetic basis of stroke and its comorbidities (strokegenetics.org). It was formed in 2007 by a small group of scientists and has since grown to over 200 members representing over 50 countries in North and South America, Europe, Australia, Asia, and Africa. ISGC workshops are held semi-annually and provide a forum for ISGC scientists and researchers to report on progress of ongoing scientific projects and discuss new ideas that help advance the ISGC's research mission. Herein, we present the Proceedings and official published abstracts of the 23rd Workshop of the ISGC, which was the first ISGC Workshop held in Asia.

At the 23rd ISGC Workshop many important ISGC themes were followed-up including the results of the latest genomic studies of intracerebral hemorrhage, intracranial aneurysm, and ischemic stroke. Several novel research topics including stroke-related metabolomics, brainimaging and epigenomics were presented. The Consortium is actively expanding its scope from European and African ancestry to other ethnic groups worldwide, additionally, welcoming new members from several Asian Biobanks, including from Japan, Korea, China, and Taiwan during this $23 r d$ workshop.

On behalf of the ISGC Steering Committee and its many members, we hope that you enjoy reviewing our Proceedings and we welcome any opportunities for new collaborations.

Acknowledgment: Steering Committee-Stephanie Debette, Chair and Jin-Moo Lee, Vice Chair; Data Access Committee-Jemma Hopewell; Analysis Committee-Rainer Malik; Outreach Committee-Jenny Majersik; Acute Endophenotypes Working Group-Jin-Moo Lee and Israel Fernandez; Imaging Working Group-Natalia Rost; Outcomes Working Group-Arne Lindgren; Intracerebral Hemorrhage Working Group-Jonathan Rosand and Daniel Woo; Intracranial Aneurysm Working Group-Ynte Ruigrok; Translational Science Working Group-Chris Anderson; Biobank Access Working Group-Jemma Hopewell.

Correspondence Dr. Kamatani kamatani.yoichiro.7s@kyoto-u.ac.jp 


\section{Brain imaging phenotypes in UK biobank}

Hideaki Suzuki, $M D, P h D^{1}$ and Paul M. Matthews OBE, $M D$, DPhil, FRCP, FMedSci ${ }^{1,2}$

${ }^{1}$ Division of Brain Sciences, Department of Medicine, Hammersmith Hospital, Imperial College London; and ${ }^{2} \mathrm{UK}$ Dementia Research Institute at Imperial College London, UK.

Objective To introduce possibly useful brain imaging phenotypes in UK Biobank. Background UK Biobank recruited about 500,000 community-dwelling participants aged 40-69 years across Great Britain between 2006 and 2010. Since 2014, a subset of participants started to acquire imaging assessment and $\sim 10,000$ of brain MRI, including T1-weighted structural, resting-state functional, task functional, T2weighted FLAIR structural, diffusion, and susceptibilityweighted structural imaging, have been collected so far. Design/Methods Details of the brain MRI image acquisition are available online. As an example of brain MRI results, we showed differences in brain volume and white matter microstructures, which were derived from $\mathrm{T} 1$-weighted structural and diffusion imaging, respectively, between hypertensives (either SBP more or equal $140 \mathrm{~mm} \mathrm{Hg}$, DBP more or equal $90 \mathrm{~mm} \mathrm{Hg}$, or antihypertensives) and non-hypertensives $(\mathrm{N}=1,559$ each $)$ and between pre-hypertensives (SBP $120-139$ and DBP $80-89 \mathrm{~mm} \mathrm{Hg}$ ) and normotensives ( $\mathrm{SBP}<120 \mathrm{~mm} \mathrm{Hg}$ and $\mathrm{DBP}<80 \mathrm{~mm} \mathrm{Hg}$ ) $(\mathrm{N}=694$ each). Results Hypertensives showed lower hippocampal volume, lower axonal density and higher periaxonal water in several white matter tracts including the superior longitudinal fasciculus (red arrows) and the superior thalamic radiation (blue arrows) relative to nonhypertensives. The periaxonal water difference in the superior longitudinal fasciculus and the superior thalamic radiation (orange and light blue arrows) were found also between pre-hypertensives and normotensives. Conclusions Brain imaging phenotypes in UK Biobank provide useful endophenotypes for late life diseases.

Contact Email: h.suzuki@imperial.ac.uk

Disclosure and Study Support: H.S. received Research Fellowship from the Uehara Memorial Foundation. P.M.M. thanks the Edmond J. Safra Foundation and Lily Safra, the UK Dementia Research Institute, and MRC funding for the Dementias Platforms UK. We have no conflict of interest for this presentation.

\section{Profiles and interests of Samsung Medical Center stroke research: New participant of ISGC}

J.W. Chung, MD, MSc, ${ }^{1}$ H.H. Won, $M D, P h D,{ }^{2}$ W.K. Seo, $M D$, $P h D,{ }^{1}$ O.Y. Bang, $M D, P h D,{ }^{1}$ and G.M. Kim, $M D, P h D^{1}$

${ }^{1}$ Department of Neurology, Samsung Medical Center, Sungkyunkwan University School of Medicine, Korea; and

\section{Axonal density}
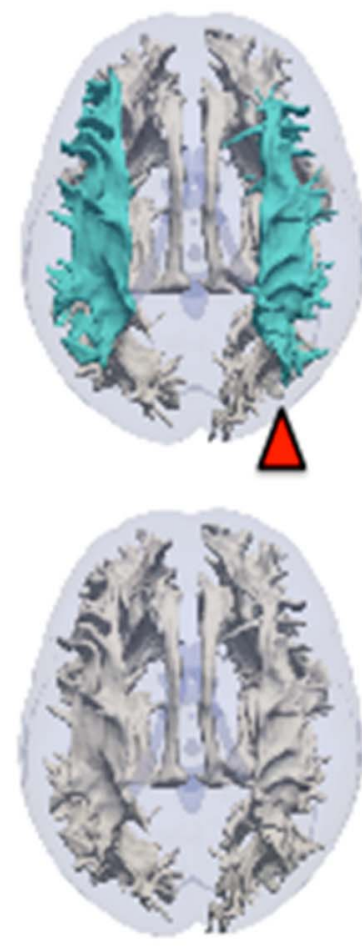
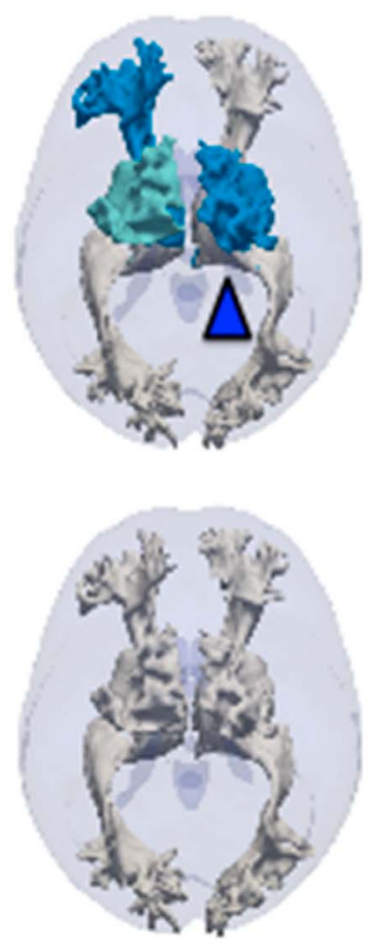

\section{Periaxonal water}
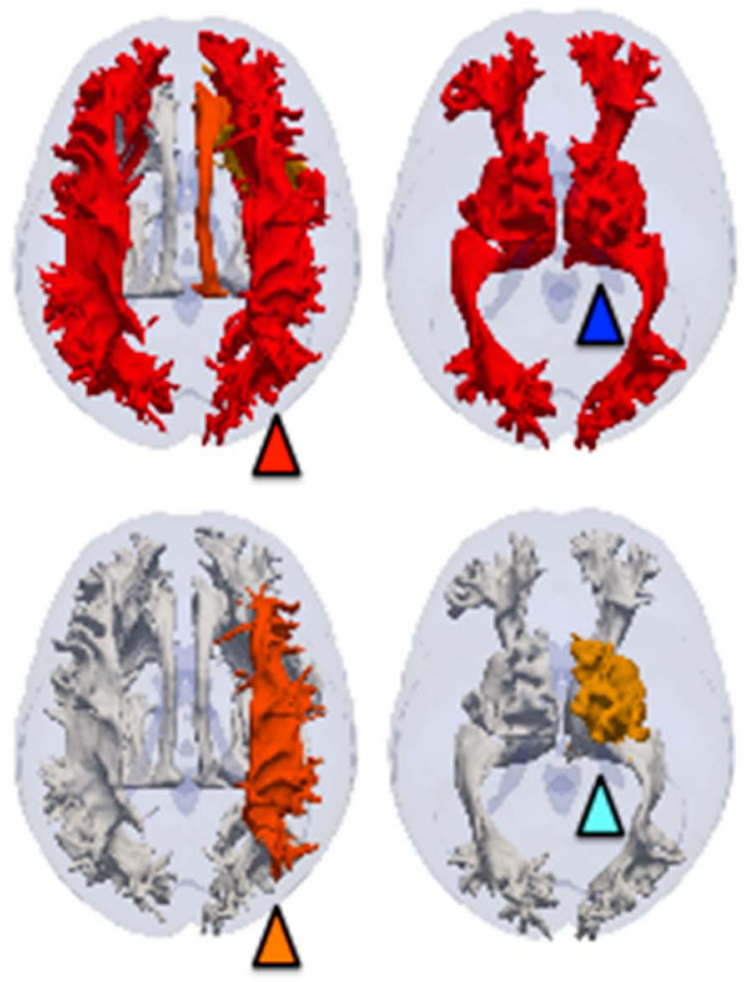
${ }^{2}$ Samsung Advanced Institute for Health Sciences and Technology, Sungkyunkwan University, Samsung Medical Center, Seoul, Republic of Korea.

Objective To introduce current general and genetic research items ongoing at Samsung Medical Center (SMC) to ISGC colleagues. Background Four stroke physicians (Jong-Won Chung, Woo-Keun Seo, Oh Young Bang, and Gyeong-Moon Kim) and many collaborators including neurosurgeon, neuroradiologist, computer scientist, genetic specialist, artificial intelligence experts are working together to improve diagnostic approach and therapeutic options for stroke patients. Design/Methods In SMC, diverse design of researches including, prospective registry (SMC stroke registry for back bone of general research), randomized clinical trial (ESCAPE, ESCAPE-NA1, and regional multi-center trials), translational research (stem cell and microvesicle), imaging researches (multimodal MR imaging, high-resolution $\mathrm{MR}$, and postprocessing). One unique SMC stroke research data characteristic is that blood samples of over 3,000 consecutive acute ischemic strokes (within 7 days of onset) confirmed by diffusion weighted imaging has been collected and stored with detailed clinical information (NIHSS scores, detailed stroke subtype after extensive etiologic work-ups, and clinical outcome up to 90-days). Results Utilizing collected samples, we are currently evaluating possible genetic factors associated with moyamoya disease and poor response to high-dose statin treatment in symptomatic intracranial atherosclerosis. Fortunately, subgroup of SMC patients is now included in GENISIS study run by Jin-Moo Lee. In addition, we would like to further collaborate with ISGC members in various studies that requires detailed clinical, laboratory, and imaging information. Conclusions We believe that SMC data incorporated with ISGC's established genetic data would help ethnicity specific and more generalizable unitization of genetic information for stroke risk stratification and evaluation of treatment response.

Contact Email: neurocjw@gmail.com

\section{Disclosure and Study Support: None.}

\section{Genome-wide association studies for subtypes of stroke in a Han Chinese population}

Tai-Ming Ko, PhD, ${ }^{1}$ Tsong-Hai Lee, MD, PhD, ${ }^{2}$ Chien-Hsiun Chen, PhD, ${ }^{1}$ Yuan-Tsong Chen, $M D, P h D,{ }^{1}$ and Jer-Yuarn $W u$, $P h D^{1}$

${ }^{1}$ Institute of Biomedical Sciences, Academia Sinica, Taipei; and ${ }^{2}$ Chang Gung Memorial Hospital, Linkou Medical Center, and Chang Gung University College of Medicine, Taoyuan, Taiwan.

Objective Because the etiology of stroke is heterogeneous, we hypothesize that genetic factors may vary by etiologic subtypes or ethnicities. Thus, we aim to identify genetic factors that contribute to 2 subtypes of stroke: hypertensive intracerebral hemorrhage $(\mathrm{HICH})$ and intracranial large-artery atherosclerosis (I-LAA). Background Although family history studies in stroke support that genetic factors may be involved in the pathogenesis, it is still unclear which particular genetic factors contribute to HICH or I-LAA. Design/Methods For HICH, a genome-wide association study (GWAS) was conducted on 363 individuals with $\mathrm{HICH}$ stroke and 1,731 controls from a Han Chinese population residing in Taiwan. The study was replicated in an independent Han Chinese population comprising an additional $250 \mathrm{HICH}$ stroke cases and 1,265 controls. For I-LAA, a GWAS was conducted on 170 individuals with I-LAA stroke and 1,731 controls from a Han Chinese population residing in Taiwan. Results In the HICH GWAS, 3 SNPs clustered at chromosome 1 , with $p$ values lower than $1 \times$ $10^{-6}$, were identified. Other 5 loci at chromosome 8,15 , and 21 were also identified in the independent Han Chinese population. In the I-LAA GWAS, 3 SNPs clustered at chromosome 10 , with $p$ values lower than $1 \times 10^{-5}$, were identified. Conclusions Genome-wide association studies conducted in a Han Chinese population could be helpful for understanding of the genetic basis of $\mathrm{HICH}$ and I-LAA.

Contact Email: jywu@ibms.sinica.edu.tw

Disclosure and Study Support: Academia Sinica Genomic Medicine Multicenter Study, Taiwan (40-05-GMM) and Chang Gung Memorial Hospital (BMRP 274, CMRPG35072, CMRPG 35073 and CMRPG 39082). There is no conflict of interest.

\section{Tohoku Medical Megabank Project Community-Based Cohort Study}

Atsushi Hozawa, $\mathrm{MD}, \mathrm{PhD}^{1}$ and Kozo Tanno, $\mathrm{MD}, \mathrm{PhD}^{2}$

\footnotetext{
${ }^{1}$ Tohoku Medical Megabank Organization, Tohoku University; and ${ }^{2}$ Iwate Medical Megabank Organization, Iwate Medical University.
}

Objective It aimed to facilitate solutions to the medical problems in the aftermath of the Great East Japan Earthquake by introducing personalized medicine and health care in the damaged areas. Background The Tohoku Medical Megabank Project Community-Based Cohort Study (TMM CommCohort Study), part of the TMM Project in Japan, was established in 2013. It was developed as an ongoing project to integrate data on various factors in the community population. Design/Methods Between May 2013 and March 2016, community residents were recruited to this study. The baseline survey consisted of self-administered questionnaires on demographic data, health habits, and psychological factors. Urine specimens and blood samples were collected, and DNA was extracted from the blood samples. To perform a more detailed assessment of the physiologic health status of the 
subjects, several additional physiologic tests, including home blood pressure measurement, intima-media thickness, and so on were conducted. Results Overall, we recruited 117,886 community residents for inclusion in this study, of which 87,167 residents agreed to be enrolled. The mean (SD) age of the subjects was $61.6(11.5)$ and 58.5 (12.2) years for men and women, respectively. Compared with participants lived in inland area, participants lived in the coastal area showed higher risk of mental damage, higher rate of withdrawal from antihypertensive medication, and higher prevalence of metabolic syndrome. These differences might yield higher rate of stroke incidence and mortality in the Tsunami affected area. To avoid the secondary health damage, we will follow-up participants' subclinical atherosclerosis to clarify the risk factors for stroke. The secondary survey recently began in June 2017. Conclusions This study, having overviewed the rationale for initiating the TMM CommCohort Study, is important for examining the pathogenesis of personalized medicine and health care among individuals in the areas affected by the Great East Japan Earthquake.

Contact Email: hozawa-thk@umin.ac.jp

Disclosures: None.

\section{Blood pressure traits differentially affect risk of different ischaemic stroke subtypes}

D. Gill, $M D$

Imperial College London, School of Public Health, Department of Epidemiology and Biostatistics, London, United Kingdom.

Objective The aim of this study was to use Mendelian randomization to estimate the causal effect of systolic, diastolic and pulse pressure of risk of different ischaemic stroke subtypes. Background Although blood pressure is an established risk factor for ischaemic stroke, its effect on different disease subtypes is not known. Observational studies in this area are limited by confounding, and randomized controlled trials are often unfeasible. Such limitations can be overcome using Mendelian randomization, which employs genetic variants as proxies for the effect of different blood pressure traits. Design/Methods A 2-sample Mendelian randomization approach was used. Instruments and genetic association estimates for blood pressure taken from a genetic association study in over 1 million people of European ancestry. Genetic association estimates for ischaemic stroke subtypes were taken from a genetic association study of approximately 16,851 stroke cases and 32,473 controls. Appropriate statistical sensitivity analyses were used to explore and adjust for any pleiotropic effect of the genetic variants on risk of stroke risk through pathways independent of blood pressure.
Results For risk of large artery stroke and small vessel stroke, Mendelian randomization evidence supported a causal effect of systolic, diastolic and pulse pressure. This was greater in magnitude than that observed for coronary artery disease. In contrast, no causal effect on risk of cardio-embolic stroke was identified for any blood pressure trait. Conclusions Blood pressure traits have a causal effect on the large artery and small vessel subtypes of ischaemic stroke and should be targeted in these diseases.

Contact Email: dipender.gill@imperial.ac.uk

Disclosure and Study Support: D.G. is funded by the Wellcome Trust.

\section{The Geisinger MyCode Community Health Initiative: A unique biobank for precision health}

Ramin Zand, MD, MPH, ${ }^{1}$ Vida Abedi, PhD, ${ }^{2}$ Christoph J. Griessenauer, $M D,{ }^{1}$ Thomas N. Person, MS, ${ }^{2}$ Neil Martin, MD, ${ }^{1}$ and David J. Carey $\mathrm{PhD}^{3}$

${ }^{1}$ Neuroscience Institute, Geisinger Health System; ${ }^{2}$ Biomedical and Translational Informatics Institute, Geisinger Health System; and ${ }^{3}$ Weis Center for Health Research, Geisinger Health System, Danville, PA.

Objective The goal of this preliminary analysis is to highlight the potentials of Geisinger MyCode initiative to study neurogenomic aspects of stroke and other cerebrovascular diseases. Background Unlike personalized medicine, precision health underscores an innovative prevention and treatment approach based on each patient-specific characteristic including genetics and clinical biomarkers. The initiation of next-generation sequencing has paved the way for accelerated genomic discoveries and is bringing precision health into the daily medical practice. Geisinger Health System is a large integrated health system located in north central and northeastern Pennsylvania. Geisinger includes a large primary care and specialty group practice in more than 70 care sites including 5 tertiary care teaching hospitals, an insurance operation, and a medical school. Design/Methods Geisinger serves a population of more than 3 million with a low rate of migration $(<1 \%$ drop-out rate) and many multigeneration families. Geisinger was one the first sites which fully implemented electronic health record (EHR) across all care sites. The system later formed an enterprise data warehouse that consolidates data from EHR and other sources and a project now known as the MyCode Community Health Initiative (MyCode) to create a platform for precision health. As of the Summer of 2017, the genomic data from more than 90,000 MyCode participants have been analyzed and available for research and targeted preventive care. Results Our preliminary analysis indicates that in the MyCode cohort 
approximately 10,000 have a diagnosis of cerebrovascular occlusive disease, stroke, or transient ischemic attack. Approximately 5,000 patients have a diagnosis of cerebral aneurysms or subarachnoid hemorrhage. In total there are close to 20,000 individuals in MyCode with an MRI of the brain. Conclusions The MyCode cohort represents a unique biobank to discover and validate neurogenomic aspects of stroke and other cerebrovascular diseases.

Contact Email: ramin.zand@gmail.com

\section{Disclosures: None.}

\section{Genetic imbalance affects functional outcome after ischemic stroke}

John W. Cole, MD, MS, ${ }^{1}$ Kristina Schlicht, PhD, ${ }^{2}$ Dorothea Pfeiffer, $M D,{ }^{3}$ Didier Leys, $M D,{ }^{4}$ Stephanie Debette, $M D,{ }^{5}$ Alessandro Pezzini, MD, ${ }^{6}$ Stefan Engelter, MD, ${ }^{7,10}$ Philippe Lyrer, $M D,{ }^{7}$ Turgut Tatlisumak, $M D,{ }^{8,11}$ Steven J. Kittner, $M D, M P H,{ }^{1}$ Arne Lindgren, $\mathrm{MD}, \mathrm{PhD},{ }^{9}$ and Caspar Grond-Ginsbach, $\mathrm{PhD}^{3}$

${ }^{1}$ Department of Neurology, Veterans Affairs Medical Center and Department of Neurology, University of Maryland School of Medicine, Baltimore; ${ }^{2}$ Institute of Medical Statistics and Informatics, Kiel University; ${ }^{3}$ Department of Neurology, University of Heidelberg, Germany; ${ }^{4}$ Department of Neurology, University of Lille; ${ }^{5}$ Department of Public Health, Bordeaux University Hospital, France; ${ }^{6}$ Department of Clinical and Experimental Sciences, Neurology Clinic, University of Brescia, Italy; ${ }^{7}$ Department of Neurology and Stroke Center, University Hospital Basel, Switzerland; ${ }^{8}$ Department of Neurology, Sahlgrenska University Hospital, Gothenburg; ${ }^{9}$ Department of Clinical Sciences, Lund, Neurology, Lund University, Sweden; ${ }^{10}$ Neurorehabilitation Unit, University Center for Medicine of Aging and Rehabilitation Basel, Felix Platter Hospital, University of Basel, Switzerland; and ${ }^{11}$ Department of Neurology, Helsinki University Central Hospital, Helsinki, Finland.

Objective To examine if genetic imbalance is associated with functional outcome after ischemic stroke. Background Genetic imbalance occurs when a protein-coding gene has more or fewer copies than the 2 copies of a normal diploid genome. Such imbalance has been associated with various disease phenotypes. Ohnologs are genes with pronounced dose-sensitivity. DESIGN/METHODS We explored the impact of genetic imbalance with and without ohnologs on outcome after ischemic stroke (IS) in 2 independent study samples using logistic regressions. Copy number variation $(\mathrm{CNV})$ was detected by PennCNV analysis of GWAS-microarrays. CNV Findings were individually validated after noise reduction1. Genetic imbalance was studied in IS patients with favorable ( $\mathrm{mRS} 0-2$ ) and unfavorable (mRS 3-6) outcome 3 months after stroke from the CADISP study ( $\mathrm{n}=816$; age $=44 \pm 10$ years $)$. To validate the findings, similar analyses were performed in IS patients from 7 SiGN/GISCOME centers $(n=2,498$; age $=68 \pm 14$ years). Genetic imbalance was analysed as a continuous variable by counting the number of imbalanced protein-coding genes per patient. Models were adjusted for age, sex, stroke severity (NIHSS), stroke subtype (TOAST) and ancestry-informative principal components. Results The number of imbalanced genes was negatively associated with favorable outcome in CADISP $(p=0.001$; OR $=0.89 ; 95 \% \mathrm{CI}$ $=0.82-0.95)$ as well as in SiGN/GISCOME $(p=0.003$; OR = $0.94 ; 95 \% \mathrm{CI}=0.91-0.98)$. Upon subgroup analysis, Imbalance affecting ohnologs was negatively associated with good outcome in the SiGN/GISCOME sample ( $p=0.002$; OR = 0.93; $95 \% \mathrm{CI}=0.89-0.98)$ as well as in the CADISP sample ( $p=$ 0.002 ; $\mathrm{OR}=0.88 ; 95 \% \mathrm{CI}=0.80-0.95)$. In contrast, such association was lacking for imbalances without ohnologs in both studies (SiGN/Giscome $p=0.9$, CADISP $p=0.4$ ). Conclusions Genetic imbalance is an independent predictor of unfavorable outcome 3 months after stroke.

Contact Email: JCole@som.umaryland.edu

Disclosure and Study Support: Presented on behalf of the CADISP (Cervical Artery Dissections and Ischemic Stroke Patients), SiGN, GISCOME (Genetics of Ischaemic Stroke Functional Outcome) studies and the International Stroke Genetics Consortium ISGC. Dr Cole was partially supported by National Institutes of Health (NIH) grants R01NS100178 and R01-NS105150, the US Department of Veterans Affairs, the American Heart Association (AHA) Cardiovascular Genome-Phenome Study (Grant-15GPSPG23770000), and the AHA-Bayer Discovery Grant (Grant-17IBDG33700328).

\section{Biobank Japan}

\section{Yoichiro Kamatani, $\mathrm{MD}, \mathrm{PhD}^{1,2}$}

${ }^{1}$ Center for Genomic Medicine, Kyoto University Graduate School of Medicine; and ${ }^{2}$ Laboratory for Statistical Analysis, RIKEN Center for Integrative Medical Sciences.

Objective Introduce the achievements and data availability of Biobank Japan. Background Biobank Japan (BBJ) was established in 2003 to collect DNA sample, serum sample and clinical information affected for 47 diseases. DESIGN/ METHODS BBJ is a Hospital-based biobank. Patients were recruited from 12 core hospitals and their branches, with collected DNA sample, serum sample and clinical information attained for over 200,000 Japanese patients. Whole-genome SNP genotyping was performed for most participants, and whole-genome sequencing for a subset. Results Currently, BBJ is the largest non-European genomic cohort with wholegenome SNP data available. Using the SNP genotypes, we performed GWAS for 44 diseases, 58 quantitative traits, and 30 life-style factors. This yielded identification of hundreds of novel susceptibility loci, indicating the utility of non-European genetic samples. The published summary statistics are now available at our jenger website (jenger.riken.jp/en/). Some of 
the traits have already been included in the international transethnic studies, including MEGASTROKE. Conclusions BBJ data should further boost genetic analyses for complex traits.

Contact Email: kamatani.yoichiro.7s@kyoto-u.ac.jp

Disclosure and Study Support: No conflict of interest. This study was funded by the BioBank Japan project, which is supported by the Ministry of Education, Culture, Sports, Sciences and Technology of Japanese government and the Japan Agency for Medical Research and Development.

\section{Genome-wide association study and meta-analysis of functional outcome after ischemic stroke}

Joint work by the GISCOME network, on behalf of the ISGC and the NINDS-SiGN Consortium. Supervised by J.M. Maguire, PhD, $R N, B A,{ }^{1} A$. Lindgren, $M D, P h D,{ }^{2}$ and C. Jern, $M D, P h D^{3}$

${ }^{1}$ University of Technology, Sydney, Australia; ${ }^{2}$ Lund University, Sweden; and ${ }^{3}$ University of Gothenburg, Sweden.

Objective The aim of the study is to exploratively investigate the genetic factors of ischemic stroke outcome. Background There is large variation in outcomes after ischemic stroke and genetic factors may account for part of this variation. Here we present the first results from the genome-wide association (GWA) analyses from the Genetics of Ischaemic Stroke Functional outcome (GISCOME) network. Design/Methods The GWAS includes 6,165 patients with ischemic stroke from 17 cohorts. ${ }^{1}$ The primary outcome was modified Rankin Scale (mRS) after 60-190 days, dichotomized in 2 ways (0-1 vs 2-6 and $0-2$ vs 3-6). We also performed ordinal analysis of $m R S$ scores. GWA analyses were performed in each cohort separately and results were meta-analyzed using a fixed effects model with inverse variance weights. Analyses were adjusted for age, sex, stroke severity (baseline NIHSS), and the 5 first principal components. Results An intronic variant (rs1842681) in the LOC105372028 gene was associated with outcome defined as mRS $0-2$ vs $3-6(p=6.8 \times 10-9)$. LOC105372028 is highly expressed in the brain, but its function remains to be elucidated. We also found several variants suggestively associated with outcome $\left(p<10^{-5}\right)$. For example, an intronic variant (rs78734480) in the NTN4 gene was suggestively associated $\left(\mathrm{P}=1 \times 10^{-6}\right)$ with $\mathrm{mRS}$ in the ordinal analysis. NTN4 has been reported to influence post-stroke functional recovery in mice. Conclusions In conclusion, we performed the largest GWAS on stroke outcome to date identifying one genome-wide significant locus and some suggestive variants implicating potential mechanisms in stroke outcome.

1. Maguire JM, et al. GISCOME-Genetics of ischaemic stroke functional outcome network: a protocol for an international multicentre genetic association study. European Stroke Journal 2017;2:229-237.

Contact Email: erik.lorentzen@gu.se

Disclosures: None

\section{Improved genomic coverage of SiGN and MRI-GENIE subjects with re-imputation using HRC reference genome}

H. Xu, MD, PhD, ${ }^{1}$ B. Gaynor, BS, ${ }^{1}$ T.J. Jaworek, BS, ${ }^{1}$ M. Lynch, BS, ${ }^{1}$ K.A. Ryan, MS, ${ }^{1}$ A.K. Giese, $M D,{ }^{2}$ J.W. Cole, MD, ${ }^{1,3}$ S.J. Kittner, MD, MPH, ${ }^{1,3}$ N.S. Rost, $M D{ }^{2}$ and B.D. Mitchell, $\mathrm{PhD}^{1,3}$; On behalf of the Stroke Genetics Network (SiGN) Consortium

${ }^{1}$ University of Maryland School of Medicine, Baltimore; ${ }^{2}$ Massachusetts General Hospital and Harvard Medical School, Boston; and ${ }^{3}$ Baltimore Department of Veterans Affairs Medical Center, MD.

Objective The objective of this report is to compare imputation coverage and quality between the $1000 \mathrm{G}$ and HRC reference panels in the Stroke Genetics Network (SiGN), an NIH-funded collaboration whose goal is to identify the genetic determinants of ischemic stroke. We hypothesize that re-imputing $\mathrm{SiGN}$ genotyping data with the HRC reference panel will increase the imputation accuracy and genomic coverage, and thus can improve our power for discovering novel genetic loci for the SiGN and embedded MRI-GENIE (MRI-Genetics Interface Exploration) projects. Background Modern genome-wide association studies (GWAS) rely on high density SNP arrays and an imputation step to increase coverage of the genome even further to enable comprehensive interrogation of genetic variation across the genome. Until recently, the 1000 Genomes $(1000 \mathrm{G}$ ) resource has served as the standard imputation reference panel for haplotype diversity. $\mathrm{Re}$ cently, the Haplotype Reference Consortium (HRC) has replaced $1000 \mathrm{G}$ as the standard. The HRC combines data sets from 20 different studies, including $1000 \mathrm{G}$, and includes 39,235,157 SNPs that define 64,976 haplotypes. In contrast, the $1000 \mathrm{G}$ (phase 3) reference panel includes $>88$ million variants although these define only 5,008 haplotypes, It has been shown that the HRC reference panel can provide accurate genotype imputation at minor allele frequencies as low as $0.1 \%$, as opposed to MAF of $\sim 1 \%$ for $1000 \mathrm{G}$, thus increasing genomic coverage and accuracy for association testing. DESIGN/METHODS Following stringent quality control filtering, SiGN GWAS array data was uploaded to the Michigan Imputation Server (imputationserver.sph.umich.edu/) for imputation against both the $1000 \mathrm{G}$ and HRC (version r1.1) panels. Eagle v2.4 
and Minimac3 were used for Phasing and imputation, respectively. Quality control analysis was performed post imputation. Results Imputation diagnostics demonstrated overall high-quality imputation with both panels. We applied imputation quality score $\mathrm{R}^{2} \geq 0.5$ to retain high quality imputed variants. Overall, the HRC panel resulted in a $36.6 \%$ increase in the total number of high imputation quality variants. For variants with MAF $<1 \%$, the HRC panel leads to an $86.0 \%$ increase in the number of highquality imputed variants, although it also results in a $20.0 \%$ decrease of imputed variants with $\mathrm{MAF} \geq 1 \%$. Conclusions The use of HRC reference panel for GWAS imputation substantially increases genomic coverage for rare variants compared to $1000 \mathrm{G}$. However, the number of imputed common variants decreased. It may be better to combine the imputed data after imputing with each reference panel alone.

Contact Email: hxu@som.umaryland.edu

Disclosure and Study Support: Supported by NIH grants (1R01NS100178, R01NS086905, and 5K23NS064052).

\section{Lateralization of acute ischemic stroke on DWI-MRI in the MRI- GENIE study}

Mattias Drake, MD, ${ }^{1}$ Björn M Hansen, MD, PhD, ${ }^{1}$ Petrea Frid, $M D,{ }^{1}$ Arne Lindgren, $M D, P h D,{ }^{1}$ Ona $W u, P h D,{ }^{2}$ Natalia Rost, $M D, P h D,{ }^{2}$ and Johan Wasselius, $M D, P h D,{ }^{1}$ for the MRI-GENetics Interface Exploration (MRI-GENIE) study investigators

${ }^{1}$ Lund University, Skåne University Hospital, Sweden; and ${ }^{2}$ Massachusetts General Hospital, Harvard Medical School, Boston.

Objective To test if left-sided lesions is more common in a large international cohort of patients with AIS and standardized MRI assessments. Background Left-sided lesions in acute ischemic stroke (AIS) has been suggested to be more common than right-sided lesions in previous hospital-based studies while a recent population-based study utilizing MRI did not to show any differences in lateralization. Design/Methods The MRI-Genetics Interface Exploration (MRI-GENIE) study is an international collaboration combining genetic data and highquality radiologic phenotype data to facilitate new discoveries in AIS genetics. Of 3,318 patients with AIS (38.9\% female; age 62.4 years \pm 14.7 ) from 12 centers in MRIGENIE 2,757 patients had DWI-MRI. A core lab consisting of 2 neuroradiologists determined the lesion location on DWI-MRI according to anatomical location and vascular territory, using a standardized assessment form. Results The majority of patients with AIS and DWI-MRI had cortical/subcortical infarcts $(\mathrm{N}=1,433,52.0 \%)$, while
$404(14.6 \%)$ had basal ganglia infarcts, 369 (13.4\%) had infratentorial infarcts, 281 (10.2\%) had multiple infarct locations, and 270 (9.8\%) had no visible infarct on DWIMRI. Left-sided lesions were more common $(\mathrm{N}=1,188,53 \%)$ than right-sided lesions $(\mathrm{N}=1,070,47 \% ; p=0.013)$ when excluding 228 patients with bilateral ischemic lesions and the 270 patients with no visible lesion. In the subset of patients with anterior circulation lesions the distribution was identical with $856(53 \%)$ left sided and 759 (47\%) right sided lesions ( $p=$ 0.016 ). Conclusions To conclude, left sided ischemic lesions are more common than right sided lesions in a large international cohort of patients with AIS, indicating an underlying pathophysiologic mechanism or differences in clinicians' abilities to detect right-sided AIS.

Contact Email: johan.wasselius@gmail.com

Disclosure and Study Support: None.

\section{Lipidomic risk factors associated with the ischemic strokes and coronary artery diseases: A Mendelian randomization approach}

S.J. Lee, $M P H, P h D,{ }^{1}$ J.Y. Ahn, BS, ${ }^{1}$ and J. Sung, $M D, P h D^{1}$

${ }^{1}$ Seoul National University School of Public Health, Korea.

Objective To identify lipid metabolites which are causally associated with ischemic stroke and coronary heart diseases. Background Dyslipidemia is a well-established risk factor of both ischemic stroke (IS) and coronary artery disease $(\mathrm{CAD})$. Although IS and CAD share many risk factors, it is not fully understood whether similar lipids are causally associated with either or both of CAD and IS. Design/Methods An NMR-based lipidomics analysis was performed for 1,000 healthy Korean adults ( $\sim 230$ metabolites) with full genomic and epidemiologic information. We performed a genome-wide scan for all metabolites and replicated the same lipidomics-GWAS results of $>20,000$ Caucasians. We systematically identified genetic instrumental variable (IVs) for each metabolite as following: (1) SNPs/SNVs having more than moderate significance $(p<0.001)$ in Caucasians $(2)$ and replicated in the Korean studies $(p<0.01)$. We examined metabolites' IVs for their associations with the IS (Metastroke) with CCS subtypes, and also in CAD (using GWAS results (CardiOgramPlusC4D) - a Mendelian Randomization approach. We compared associated lipid profiles between IS and CAD (at $p=0.01$ level). Results A total of 114 lipid metabolites (220 Korean and 140 metabolites in Caucasian study) had more than one replicated IVs. For CAD 64 metabolites showed significant associations whereas only 11 metabolites for IS (all IS). Notably, all the metabolites causally associated with IS were also associations with 
CAD. Lipid metabolites associated with all types of IS included IDL (total and free cholesterol), large HDL (particle concentration, total and esterified cholesterol, total and phospho-lipids), small VLDL (free cholesterol, total and phospho-lipids), and total triglycerides level. Conclusions Our findings suggest that dyslipidemia is associated with IS, but with less extent than CAD. Additionally, for IS, serum
VLDL and IDL along with serum TG might have more important roles than LDL lipids.

Contact Email: jsung@snu.ac.kr

Disclosure and Study Support: We do not have any conflict of interest to disclose for this study. 


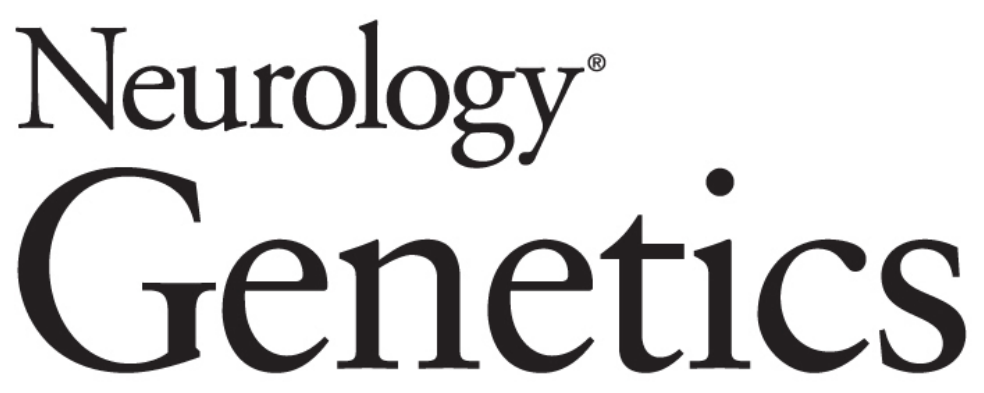

Proceedings of the 23rd International Stroke Genetics Consortium Workshop: Kyoto,

Yoichiro Kamatani and Stéphanie Debette

Neurol Genet 2019;5;

DOI 10.1212/NXG.0000000000000324

This information is current as of April 8, 2019

Updated Information \&

Services

Permissions \& Licensing

Reprints including high resolution figures, can be found at: http://ng.neurology.org/content/5/1_Supplement_1/e324.full.html

Information about reproducing this article in parts (figures,tables) or in its entirety can be found online at:

http://ng.neurology.org/misc/about.xhtml\#permissions

Information about ordering reprints can be found online:

http://ng.neurology.org/misc/addir.xhtml\#reprintsus

Neurol Genet is an official journal of the American Academy of Neurology. Published since April 2015, it is an open-access, online-only, continuous publication journal. Copyright ( 2019 American Academy of Neurology. All rights reserved. Online ISSN: 2376-7839.

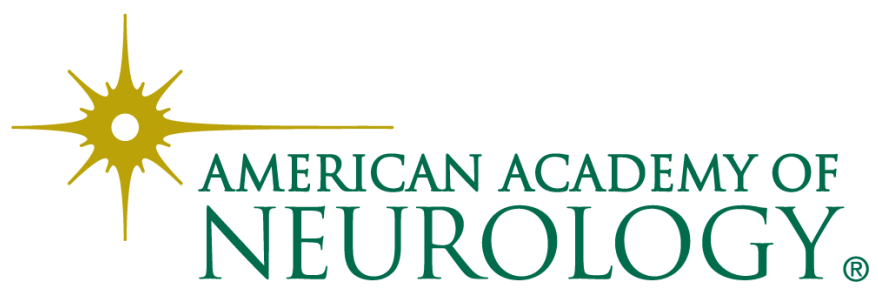

